\title{
The potential risk factors of cortical visual impairment following cerebral angiography: a retrospective study
}

This article was published in the following Dove Press journal:

Therapeutics and Clinical Risk Management

\author{
Yunna Yang' \\ Jing Zhang ${ }^{2}$ \\ Tong $\mathrm{Li}^{3}$ \\ 'Department of Neurosurgery, Beijing \\ Chao-Yang Hospital, Capital Medical \\ University, Beijing 100043, People's \\ Republic of China; ${ }^{2}$ Department of \\ Neurosurgery, Beijing Friendship \\ Hospital, Capital Medical University, \\ Beijing 100043, People's Republic of \\ China; ${ }^{3}$ Department of Interventional \\ Neuroradiology, Beijing Chaoyang \\ Hospital, Capital Medical University, \\ Beijing 100043, People's Republic of \\ China
}

Correspondence: Yunna Yang Department of Neurosurgery, Beijing Chaoyang Hospital, Capital Medical University, No. 5 Jingyuan Road, Shijingshan, Beijing 100043, People's Republic of China

Tel +86 I05 I7I 8624

Email yangyyn2015@163.com
Background: Cortical visual impairment is an uncommon complication after cerebral angiography with an incidence of $0.3 \%-1.0 \%$. Here we would like to investigate and discuss the potential risk factors of cortical visual impairment after cerebral angiography.

Methods: Based on the presence of post-operative cortical visual impairment, 4,528 patients who received cerebral angiography were split into two groups. The relevant risk factors were compared and analyzed between the groups.

Results: In the patient cohort, 11 cases exhibited post-operative cortical visual impairment (0.24\%). In particular, seven patients presented with blurred vision and four presented with binocular blindness. Visual sensation of these patients recovered after the treatment. Our univariate analysis revealed that differences in age, weight, sex ratios, proportions of patients with hypertension, diabetes and hyperlipemia, and operation time were not statistically significant between the two groups $(P>0.05)$. The multivariate analysis indicated that the dosage of contrast medium was the independent risk factor for post-operative cortical visual impairment.

Conclusion: Clinically, cortical visual impairment following cerebral angiography typically presented as blurred vision or complete blindness. We have identified the dosage of contrast medium as the most critical independent risk factor based on our study. Preventive strategies need to be implemented to avoid post-operative cortical visual impairment in this regard.

Keywords: cortical blindness, angiography, contrast agent

\section{Introduction}

Cerebral angiography, though being associated with multiple known complications, is still considered as the gold standard for diagnosing cerebrovascular diseases. ${ }^{1,2}$ Cortical visual impairment is one of the uncommon complications following cerebral angiography, myeloangiography intervention, and vascular interventional therapy at the coronary artery, aorta, brachial plexus, and brain. ${ }^{3-5}$ Transient cortical $^{-1}$ visual impairment occurs most frequently after cerebral angiography, with an incidence of $0.3-1.0 \%{ }^{4,6}$ It has been proposed to be related to the extravasation of contrast medium. ${ }^{7}$ However, the detailed etiologic mechanism of cerebral angiography-induced cortical visual impairment remains unclear. The aim of this retrospective study was to investigate possible risk factors of cortical visual impairment caused by cerebral angiography. 


\section{Materials and methods}

\section{Patient information and clinical data}

In the Department of Neurosurgery at Capital Medical University-affiliated Beijing Chaoyang Hospital and Beijing Friendship Hospital, 4,528 cases of cerebral angiography were performed between 2014 and 2018. This retrospective study was approved by the Ethical Committee of the Beijing Chaoyang Hospital. We included patients who have received cerebral angiography in the study, and collected all the relevant clinical information and past medical history from the enrolled patients, including gender, age, weight, history of diabetes, hypertension and hyperlipidemia, the dosage of contrast medium, and operation time.

The diagnostic criteria for cortical visual impairment utilized in the current study are: (1) a total or partial loss of vision and visual sensation, (2) a loss of eyelid closure reflection upon strong light or objects that are rapidly moving close, (3) preservation of the abilities to perceive light and/or moving objects, (4) normal retina without optic atrophy, (5) loss of reactions on electroencephalography (EEG) and no reactions to optokinetic nystagmus, and (6) normal electroretinogram (ERG) results with the abnormal visual evoked potential (VEP).

\section{Cerebral angiography}

Local anesthesia was performed using $2 \mathrm{ml}, 2 \%$ lidocaine. The femoral artery was approached by the Seldinger technique, and the 5F MP-A1catheter with iodinated contrast medium
(Ioversol injection, $320 \mathrm{mg}$ iodine $/ \mathrm{ml}$ ) were placed for bilateral vertebral arteries and internal carotid artery. Then, the $6 \mathrm{~F}$ catheter was installed for the diagnostic procedure.

\section{Statistical analysis}

SPSS (Version 22.0; IBM, New York) software was utilized to analyze the data. Chi-square test, paired $t$-test, and multivariate logistic regression analysis were performed. The threshold for statistical significance was set at $p<0.05$.

\section{Results}

In this patient cohort, 4,528 patients who met the inclusion criteria were enrolled, and 11 cases $(0.24 \%)$ exhibited cortical visual impairment after cerebral angiography, including 6 males and 5 females (Table 1). The average age of the patient cohort was $51.08 \pm 11.84$ years, ranging from 24 to 75 years. Patients who exhibited post-operative cortical visual impairment were slightly older, with an average age of $58.34 \pm 12.36$ years. However, there was no statistical significance between these two groups. The average body weight was $66.28 \pm 11.71 \mathrm{~kg}$ in the entire patient cohort, and $58.45 \pm 10.36 \mathrm{~kg}$ in the patients with cortical visual impairment. Blurred vision was present in 7 cases of post-operative cortical visual impairment (63.63\%), and the clinical symptom lasted for $8 \mathrm{hrs}$ to 6 days. Four patients presented with binocular blindness (36.37\%), lasting for 1-2 weeks (Table 2). In the current study, eight cases of cortical visual impairment occurred within 1-6 hrs and three cases within 6-24 hrs after the

Table I The univariate analysis of risk factors for cortical visual impairment (n [\%])

\begin{tabular}{|c|c|c|c|c|}
\hline Factors & & Total $(n=4528)$ & Cortical visual impairment $(n=I I)$ & $p$ \\
\hline Gender & $\begin{array}{l}\text { Male } \\
\text { Female }\end{array}$ & $\begin{array}{l}2,602 \\
1,926\end{array}$ & $\begin{array}{l}6(0.23) \\
5(0.26)\end{array}$ & 0.726 \\
\hline Age & & $51.08 \pm 11.84$ & $58.34 \pm 12.36$ & 0.205 \\
\hline Weight (kg) & & $66.28 \pm|1.7|$ & $58.45 \pm 10.36$ & 0.098 \\
\hline Diabetes & $\begin{array}{l}\text { Yes } \\
\text { No }\end{array}$ & $\begin{array}{l}1,592 \\
2,936\end{array}$ & $\begin{array}{l}5 \\
6\end{array}$ & 0.203 \\
\hline Hypertension & $\begin{array}{l}\text { Yes } \\
\text { No }\end{array}$ & $\begin{array}{l}1,487 \\
3,041\end{array}$ & $\begin{array}{l}4 \\
7\end{array}$ & 0.952 \\
\hline Hyperlipidemia & $\begin{array}{l}\text { Yes } \\
\text { No }\end{array}$ & $\begin{array}{l}1,696 \\
2,832\end{array}$ & $\begin{array}{l}4 \\
7\end{array}$ & 1.230 \\
\hline Contrast medium $(\mathrm{ml})$ & & $86 \pm 20$ & $120 \pm 25$ & $<0.001$ \\
\hline Operation time (min) & & $59 \pm 15$ & $72 \pm 20$ & 0.191 \\
\hline
\end{tabular}


Table 2 The clinical presentation of visual impairment

\begin{tabular}{|l|l|l|}
\hline Clinical symptoms & Cases & Percentage (\%) \\
\hline Blurred vision & 7 & 63.63 \\
Binocular blindness & 4 & 36.37 \\
Total & $\mathrm{II}$ & 100.00 \\
\hline
\end{tabular}

procedure. The visual sensation recovered after the treatment for all the affected patients. Patients with blurred vision recovered more quickly than binocular blindness, with the former recovered in 3-6 days and the latter more than seven days. Post-operative cerebral infarction and intracranial bleeding were not seen in this patient cohort. Representative radiographic and vsculature images were demonstrated in Figure 1.

The univariate analysis was performed to evaluate the risk factors for cortical visual impairment (Table 1). The factors with $p<0.2$ were then further assessed by the multivariate logistic regression analysis, including weight, contrast medium, and operation time. The results indicated that only the dosage of contrast medium $(p<0.001, O R$ $=1.133$, 95\% CI: $1.101-1.175)$ was the sole independent risk factor for cortical visual impairment (Table 3).

\section{Discussion}

Cortical visual impairment is defined as a complete or partial vision loss due to the cortical pathology of bilateral dorsal geniculate nuclei, internal capsule, and occipital lobes. ${ }^{8}$ The defective function is usually transient and reversible. ${ }^{8}$ Cortical visual impairment is a multi-factorial disorder and can be induced by occlusive or focal cerebrovascular diseases, trauma, occipital lobe infarction, cardiac arrest, toxic dysentery, and coronary or brain angiography. ${ }^{9-11}$ The incidence of post-operative cortical visual impairment after cerebral angiography is approximately $0.3-1.0 \%$, and $0.24 \%$ according to our results. Clinically, patients with cortical blindness may present with a complete loss of visual sensation and retinal reflection toward strong light; this may or may not be accompanied by hemidysesthesia, aphasia, and hemiplegia. ${ }^{9}$
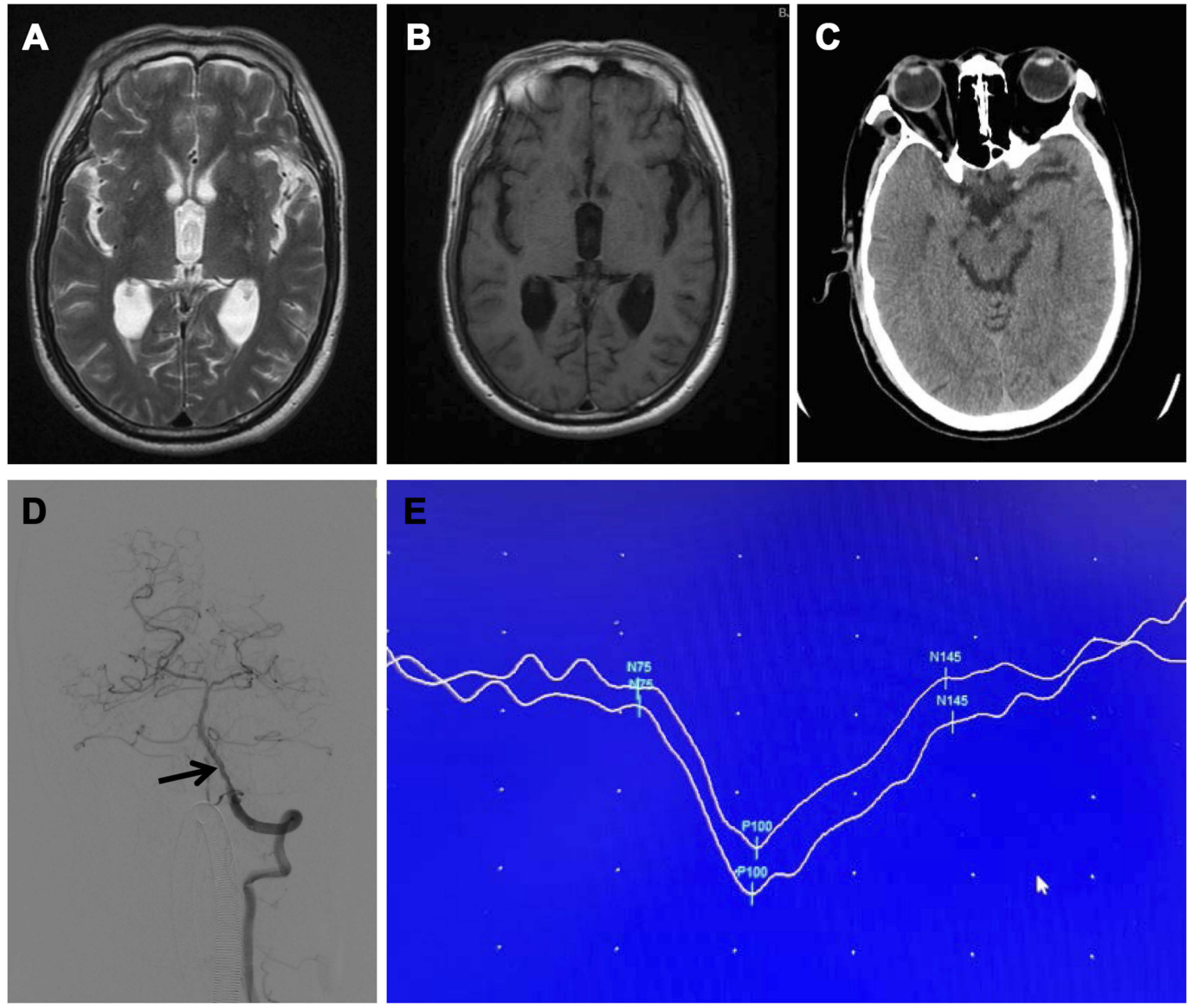

Figure I Imaging of skull and vascularity. No abnormalities of occipital lobe were identified on (A) MRI, T2, (B) MRI, TI, and (C) CT. (D) Mild vertebral stenosis was shown on the computed tomographic angiography (arrow). (E) Visual evoked potential examination demonstrated a prolonged PI00. 
Table 3 The multivariate logistic regression analysis for cortical visual impairment

\begin{tabular}{|l|l|l|}
\hline Variables & $\mathbf{P}$ & OR (95\% Cl) \\
\hline Weight $(\mathbf{k g})$ & 0.093 & $0.709(0.483-1.013)$ \\
Operation time $(\mathbf{m i n})$ & 0.175 & $1.126(0.973-1.322)$ \\
Contrast medium $(\mathrm{ml})$ & $<0.001$ & $1.133(\mathrm{I} .10 \mathrm{I}-\mathrm{I} .175)$ \\
\hline
\end{tabular}

Abbreviations: $\mathrm{OR}$, odds ratio; $\mathrm{Cl}$, confidence interval.

However, the structure of retina is within normal limit upon examination, and the pupil reflection toward light and abduction movements remain normal. ${ }^{9}$ Typically, cortical blindness occurs within six hours following the angiography procedure, and the vision recovers within several hours to days. ${ }^{9,12,13}$

The clinical studies have shown that elderly patients are more prone to post-operative cortical visual impairment. ${ }^{9}$ However, the etiopathological mechanisms are unclear. Several hypotheses have been proposed to explain this clinical condition. (A) Transient ischemia of the visual cortex caused by cerebral vasospasm: The patient may have existing cerebral vascular disorders. The injection of contrast medium changes hemodynamics, leading to vasospasm and ischemia of occipital lobe, as well as cortical visual loss. (B) The neurotoxicity of contrast medium: The extravasation of ionic contrast medium may disrupt the blood-brain barrier (BBB). In particular, contrast medium can easily infiltrate into the occipital lobe because the BBB at the area is relatively thin and fragile, which leads to cellular edema, alteration in neuronal excitability, and cortical blindness. ${ }^{14}$ Deposition of contrast medium in the occipital lobe has been demonstrated in the previous MRI study. ${ }^{15}$ Additionally, a large-scale clinical study was completed by Hung Jiang et al to investigate 2,547 patients who received cerebral angiography. The results suggested that a higher dosage of contrast medium might induce cortical blindness after procedure. ${ }^{16}$ (C) The posterior cerebral artery is the terminal branch of vertebrobasilar artery, and both posterior and middle cerebral arteries supply blood for occipital lobes. Transient ischemia or infarction of middle and posterior cerebral arteries may cause cortical blindness. It has also been reported that cortical blindness after angiography is caused by endothelin-related vasoconstriction. ${ }^{7}$ In our study, none of the patients exhibited cerebral vasospasm, and the incidence of cortical visual impairment is correlated with the dosage of contrast medium. Therefore, we prefer the theory that neurotoxicity is the main cause of cortical blindness.
Currently, there is no standard clinical protocol to treat post-operative cortical visual impairment following cerebral angiography. Early diagnosis and timely management are critical. In our center, we managed the condition by expanding cerebral arteries, reducing brain edema, and nourishing the nerves via intravenous injection $(4 \mathrm{~mL} / \mathrm{hr})$ of $10 \mathrm{mg}$ Nimodipine (Bayer Group, Germany, J20140105) with $250 \mathrm{~mL}, 20 \%$ mannitol, $500 \mathrm{~mL}$ lowmolecular dextran, and $10 \mathrm{mg}$ dexamethasone. The visual sensation of the affected patients completely recovered after our treatment regimen. A well-controlled clinical study is needed to further validate our findings.

To reduce the incidence of cortical visual impairment, it is suggested to follow the guidelines: ${ }^{14}$

1. Decrease the operation time and cut down the dosage of contrast medium.

2. In patients with existing cerebral vascular disorders, it is recommended to keep venules open and avoid cerebral vasospasm via Nimodipine.

3. Apply non-ionic contrast medium in the regular cases and carefully control the dosage.

4. Immediate $\mathrm{CT}$ and ophthalmoscope examinations should be administered if patients present with postoperative cortical visual impairment. It is critical to exclude intracranial bleeding and infarction, and provide the patients with appropriate management.

In summary, cortical visual impairment is an uncommon post-operative complication following the cerebral angiography. There are multiple risk factors associated with post-operative cortical visual impairment, and the clinical presentations are mainly blurred vision or blindness. It is critical to prevent risk factors in order to reduce the incidence of cortical visual impairment. The current study is based on the experience of two medical institutions, and due to the limited cases, the results may be biased. It is critical to collect the multi-center data for further investigation.

\section{Disclosure}

The authors report no conflicts of interest in this work.

\section{References}

1. Leow K, Murie JA. Cerebral angiography for cerebrovascular disease: the risks. Br J Surg. 1988;75(5):428-430. doi:10.1002/bjs. 1800750511 
2. Ko TH, Chiu WT, Chao YT, Chan L. Transient cortical blindness and amnesia after cerebral angiography. Neurol India. 2016;64(1):164 165. doi:10.4103/0028-3886.173659

3. Vallabhaneni R, Jim J, Derdeyn CP, Sanchez LA. Transient cortical blindness after thoracic endovascular aneurysm repair. J Vasc Surg. 2011;53(5):1405-1408. doi:10.1016/j.jvs.2010.11.101

4. Lalji A, Izbudak I, Birnbaum J. Cortical blindness and not optic neuritis as a cause of vision loss in a Sjogren's syndrome (SS) patient with the neuromyelitis optica spectrum disorder (NMOSD): challenges of ascribing demyelinating syndromes to SS: a case report. Medicine (Baltimore). 2017;96(34):e7454. doi:10.1097/ MD.0000000000007454

5. Abalo-Lojo JM, Baleato-Gonzalez S, Gonzalez F. Cortical blindness secondary to posterior reversible encephalopathy syndrome, recovered by successful blood pressure management. Arq Bras Oftalmol. 2017;80(5):324-326. doi:10.5935/0004-2749.20170079

6. Newman CB, Schusse C, Hu YC, McDougall CG, Albuquerque FC. Acute transient cortical blindness due to seizure following cerebral angiography. World Neurosurg. 2011;75(1):83-86. doi:10.1016/j. wneu.2010.07.019

7. Chen CY, Chen CJ, Tseng YC. A case report of transient cortical blindness after angiography. Neurologist. 2017;22(3):82-84. doi:10.1097/NRL.0000000000000115

8. Alp BN, Bozbuga N, Tuncer MA, Yakut C. Transient cortical blindness after coronary angiography. J Int Med Res. 2009;37(4):12461251. doi: $10.1177 / 147323000903700433$
9. Aldrich MS, Alessi AG, Beck RW, Gilman S. Cortical blindness: etiology, diagnosis, and prognosis. Ann Neurol. 1987;21(2):149-158. doi:10.1002/ana.410210207

10. Melnick MD, Tadin D, Huxlin KR. Relearning to See in Cortical Blindness. Neuroscientist. 2016;22(2):199-212. doi:10.1177/107 3858415621035

11. Cheng-Tagome S, Yamamoto A, Suzuki K, Katayama N, Imai H. Cortical blindness induced by hepatic encephalopathy: case report and review of published case reports. Acute Med Surg. 2016;4 (1):109-113. doi:10.1002/ams2.225

12. Akhtar N, Khatri IA, Naseer A, Ikram J, Ahmed W. Transient cortical blindness after coronary angiography: a case report and literature review. J Pak Med Assoc. 2011;61(3):295-297.

13. Das A, Huxlin KR. New approaches to visual rehabilitation for cortical blindness: outcomes and putative mechanisms. Neuroscientist. 2010;16(4):374-387. doi:10.1177/1073858409356112

14. Dundar NO, Aralasmak A, Gurer IE, Haspolat S. Subacute sclerosing panencephalitis case presenting with cortical blindness: early diagnosis with MRI and MR spectroscopy. Clin Neuroradiol. 2014;24 (2):185-188. doi:10.1007/s00062-013-0218-x

15. Hinchey J, Sweeney PJ. Transient cortical blindness after coronary angiography. Lancet. 1998;351(9114):1513-1514. doi:10.1016/ S0140-6736(05)78906-6

16. Jiang $\mathrm{H}$, Yang $\mathrm{W}, \mathrm{Wu}$ J. Acute transient cortical blindness occurring after cerebral angiography and vascular interventional management: a clinical analysis. J Intervent Radiol. 2013;22(1):8-11.
Therapeutics and Clinical Risk Management

\section{Publish your work in this journal}

Therapeutics and Clinical Risk Management is an international, peerreviewed journal of clinical therapeutics and risk management, focusing on concise rapid reporting of clinical studies in all therapeutic areas, outcomes, safety, and programs for the effective, safe, and sustained use of medicines. This journal is indexed on PubMed Central, CAS

\section{Dovepress}

EMBase, Scopus and the Elsevier Bibliographic databases. The manuscript management system is completely online and includes a very quick and fair peer-review system, which is all easy to use. Visit http://www.dovepress.com/testimonials.php to read real quotes from published authors 